\title{
Comparative research of diesel engine at working with anamegators
}

\author{
Georgi Komitov and D. Kehayov \\ Department of Agricultural Mechanization - Agricultural University - Plovdiv, Bulgaria
}

\begin{abstract}
In conditions of the high petrol dependence, depletion of natural resources and environmental pollution, the questions with the implementation of the various options for reducing costs in the operation of engines, and at the same time reducing the discharged into the atmosphere harmful emissions are very topical. This article looks at the work of a diesel engine running on standard diesel fuel, an anamegator for fuel and a fuel and oil anamegators. Anamagators are relatively unknown additives in our market that improve some of the engine performance. They are complex chemicals that are added to fuels and complexly improve the burning process. They applied to all liquid and gaseous fuels and all types of oils. As a result of the conducted experiments are received the dependencies of the hourly fuel consumption, the carbon dioxide, the carbon oxide, the smoke, etc. through the change of power.
\end{abstract}

\section{Introduction}

At the beginning of the 20th century, when the person was not heavily dependent on transport machines, there were different alternatives to moving and performing a certain type of activity. In the modern dynamic world it is almost impossible to carry out economic activity without transport or the use of machinery with an internal combustion engine $[1,2]$.

Even with the creation of the first internal combustion engines /engine/ are set out and the main problems for future exploitation, namely: continuous work on obtaining and ensuring their basic combustion source, and later unsuspecting in the beginning, the solution issues with environmental pollution. The pollution is with exhaust gases from their engines. The issue of optimal use of liquid fuels in industry and transport is becoming increasingly relevant. From an environmental point of view, it is important to reduce the emissions of exhaust gases. One way to achieve this effect is to reduce the fuel consumption of transport machines [3].

On the other hand, the reduction of air pollution under the Kyoto Protocol is a combination of coordinated actions of state bodies, different public organizations and each individual member of society. With such actions, using modern technologies of production in the various spheres of the economy, modernization of transport infrastructure and the realization of integration of the various modes of contemporary level will inevitably realize the objectives of a viable and a healthier lifestyle [4]. In this context, reducing fuel consumption of cars reveals one of the options for reducing air pollution from road transport [5].

Ability to reduce fuel consumption while reducing the emissions emitted by the engine is the use of various fuel additives or use other alternatives fuels from biomass [4]. Interestingly, in this case, applying these additives does not require additional adjustment of the diesel fuel system. The price for the use of these technologies is changing slightly, in contrast to the application of various new systems in diesel engines, related to reducing fuel consumption. Addition of additional additives does not require additional staff training to work with them, nor does it require any special attitude of drivers for a certain style of driving [1].

Anamegator is a proprietary name of a class of additive added to the fuel in quantities of less than $0,02 \%$ by mass and a complex fuel-enhancing process. Using of anamegators in the combustion process leads to a decrease in entropy due to the composition of the additive, and as a result increases the useful work and reducing the specific fuel consumption and toxicity of combustion products [7].

As a result of numerous tests and studies found that anamegators for oil save fuel by $15 \%$, reduce the consumption of oil in the engine a few times, reduced friction and wear of the friction surfaces, increasing the time for implementation, reduce the toxicity of exhaust gases and smoke. Oil anamagators can be used in various engine lubrication systems, regardless of the graft package used in the oils. The connections that hold the grafts in the package, when the engine is running, tear under the influence of mechanical, chemical and temperature loads. When using anamegators for the oil in the oil, the molecular and atomic bonds in the oil package become strong as possible and stability of additives in the destruction increases 3-5 times [6].

\section{Materials and methods}

Laboratory experiments were carried out in the laboratories of department "Engines and transport technique" of the "Angel Kanchev" University of Rousse. A "MS 2821-4" bench brake is used to determine engine characteristics. The „MS 2821-4“ in generator mode absorbs $100 \mathrm{~kW}$ of power at $3000 \mathrm{~min}^{-1}$, while in an electric motor mode it develops power of $83 \mathrm{~kW}$ at $3000 \mathrm{~min}^{-1}$.

The tests were conducted with a diesel engine "VAMO D-3900".

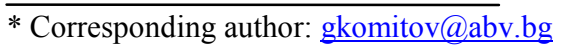




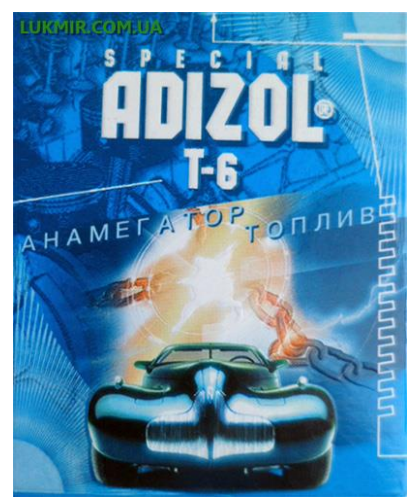

a)

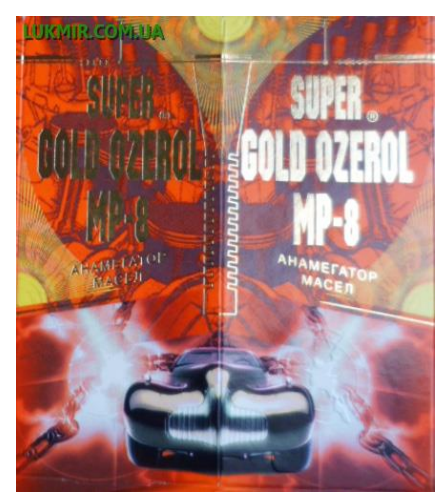

b)
Fig. 1. General appearance of anamegators: a- for fuels; $b$ - for oils.

Two types of fuel have been studied - standard diesel and mixed diesel fuel. The Mixed diesel fuel consists of $10 \%$ biodiesel (to standard diesel with 5\% biodiesel added another $5 \%$ biodiesel from rapeseed oil, production "Bulmarket" JSC - Rousse) and anamegator for fuel "ADIZOL T-6" in the ratio of $0.017 \mathrm{ml} / \mathrm{L}$ of fuel (fig. 1). The load characteristics of the engine with the two types of fuels are taken at regime $n=$ $2000 \mathrm{~min}^{-1}$. The initially, the characteristics of the engine, working only with standard diesel fuel, were taken. The fuel is then replaced with the mixed fuel with anamegator for fuel. The engine is started at a minimum speed and after a period of eight hours the engine characteristics have been taken against. In the third stage of the experiments, an anamegator for oils „GOLD OZIROL MP-8“ was added to the engine oil. The amount of anamegator for oils added is 7 $\mathrm{ml} / \mathrm{L}$ engine oil. The engine is again allowed to run for eight hours and the experiments are repeated. A heat exchanger of company "Bowman" was used to maintain a normal operating temperature.

The captured engine characteristics are loadable, since the values of the parameters studied are the most accurate. From the load characteristics, a quantitative estimate of the engine's economy when working with additives [7].

The monitored parameters are hourly fuel consumption $\mathrm{G}_{\mathrm{h}}$, smoke $\mathrm{D}$, carbon oxide $\mathrm{CO}$, carbon dioxide $\mathrm{CO}_{2}$, nitrogen oxides $\mathrm{NO}_{\mathrm{x}}$ and excess oxygen in the exhaust gases $\mathrm{O}_{2}$. In all attempts the changes in the resistance moment were reported.

Used fuel from the engine is weighed using a weighing method with a "Sartorius 524P" electronic scale with a range of $120-520 \mathrm{~g}$ and an accuracy of $0,5 \mathrm{mg}$. To determine the toxicity of exhaust gases is used a five components gazanalizer "Auto Logic plus" with accuracy $1 \%$ and the concentration of smoke is determined by opacimeter "OPA" 101 " of company "Brainbee" with precision 1\%. For measuring the time of each test is using the "JS-6619" digital timer "Junso" with an accuracy of $1 / 100 \mathrm{~s}$.

The determine the hourly fuel consumption by dependence (1) is firstly:

$$
\mathrm{G}_{\mathrm{h}}=3,6 \cdot \mathrm{G}_{\mathrm{i}} / \mathrm{t}_{\mathrm{i}}, \mathrm{kg} / \mathrm{h}
$$

where $G_{i}$ is mass of using fuel for i test, $\mathrm{kg}$;

$t_{i}$ - time for $i$ test, $s$.

After determining the hourly fuel consumption, the specific fuel consumption is re-calculated on the basis of the dependence (2):

$$
\mathrm{ge}=10^{3} \cdot \mathrm{G}_{\mathrm{h}} / \mathrm{N}_{\mathrm{e}}, \mathrm{g} / \mathrm{kWh}
$$

where $\mathrm{N}_{\mathrm{e}}$ is effective power, $\mathrm{kW}$.

\section{Experimental results}

In figure 2 shows the variation of the hourly fuel consumption depending on the effective engine power. The analyzing the figure there is a reduction in the hourly fuel consumption using mixed fuels and anamegators. In both versions - with an anamagators with fuel and anamegators for oils, the average change is within $13 \%$ compared to the standard fuel. It given the impression the almost similar characteristics and of all three charts.

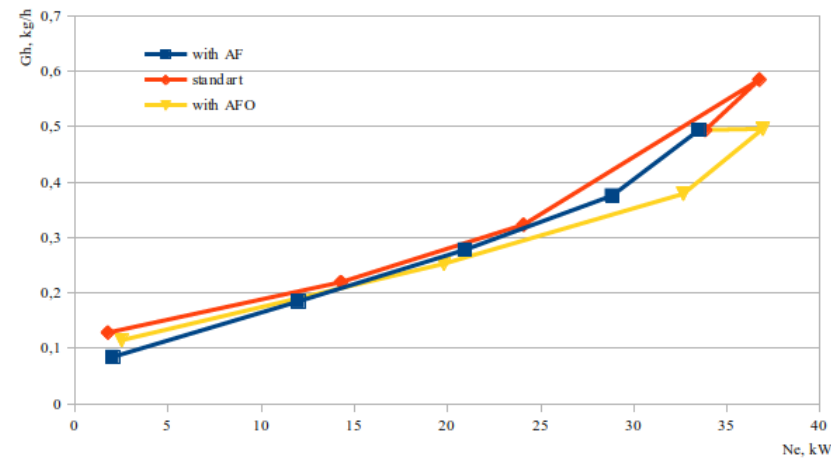

Fig. 2. Variation of hourly fuel consumption of diesel engine dependency from effective power:

with $\mathrm{AF}$ - mixed fuel with anamegator for fuels; standart - standart diesel fuel; with $\mathrm{AFO}$ - mixed fuel with anamegators for fuels and oils.

As a major benchmark for the different tests, the change in specific fuel consumption is most often used. It is the ratio of the hourly fuel consumption to the engine power. The data for this indicator is presented in figure 3 . The average value at the standard fuel is $0,023 \mathrm{~kg} / \mathrm{kWh}$, and when using a mixed fuel with an anamegator for fuel, it is $0,0189 \mathrm{~kg} /$ $\mathrm{kWh}$. When used the mixed fuel with anamegators for fuel and for oil, it is almost the same value of $0.0192 \mathrm{~kg} / \mathrm{kWh}$. Expressed in percentages this change is $21,9 \%$ for biodiesel with anamegators for oil and for fuel and $23,4 \%$ for biodiesel only with anamegators for fuel.

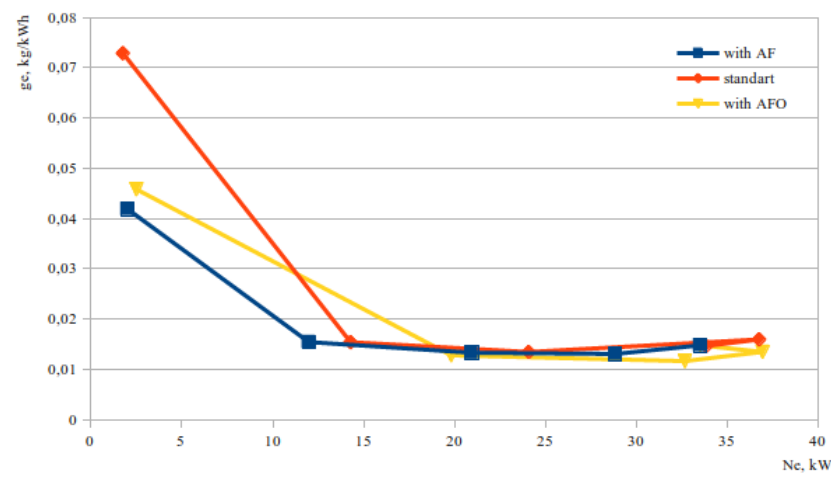

Fig. 3. Variation of specific fuel consumption of diesel engine dependency from effective power:

with AF - mixed fuel with anamegator for fuels; standart - standart diesel fuel; with $\mathrm{AFO}$ - mixed fuel with anamegators for fuels and oils.

At the chart it is noticeable that in the medium-sized loads the specific fuel consumption of the engine when using mixed fuel with anamegators for fuel and oil is the lowest and this is due to the improved quality of the oil with anamegator. The values of this indicator are almost identical 
when the fuel pump regulator is working, and at the low power the fuel consumption of both tests with mixed fuels with anamegators for fuels and mixed fuels with amanegators for oil and for fuel is significantly lower than the standard fuel.

In figure 4 shows the variation of the smoke from the exhaust gases. This is the most summary indicator of the toxicity of diesel engines. It is regulated by the legislator and monitored for its condition in all operating regimes of the engine. There is noticeable reduction in smoke when using biofuel and anamegators compared to the use of standard diesel fuel. At the figure shows that the use of standard diesel fuel gets some inertia in obtaining this indicator, especially in the sudden change of the load on the engine, due to the lower operating temperature of the gases, and consequently, the weaker the purging of the combustion chamber. Some "linearity" of the results and a slight increase of the indicator is obtained.

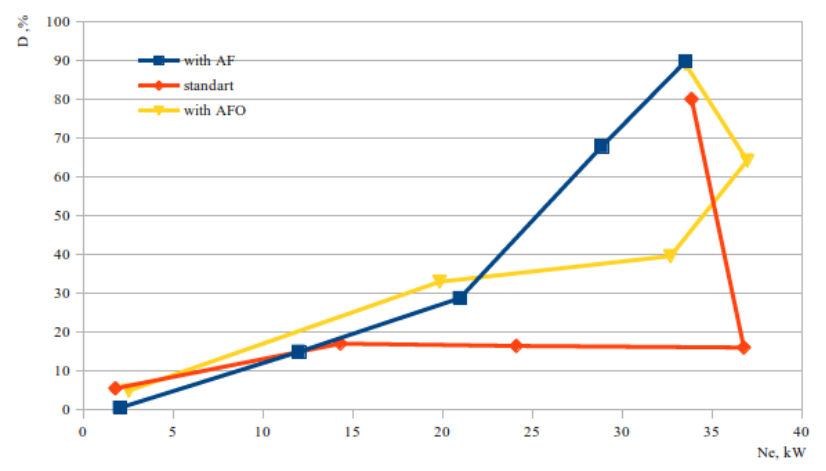

Fig. 4. Variation of smoke in the exaust gases of diesel engine dependency from effective power:

with $\mathrm{AF}$ - mixed fuel with anamegator for fuels; standart - standart diesel fuel; with AFO - mixed fuel with anamegators for fuels and oils.

The use of mixed fuel from diesel fuel, biodiesel and anamegators leads to the reduction of the highly toxic $\mathrm{CO}$ (figure5). The average reduction of this indicator is within $22 \%$ when using biodiesel with fuel anamegator for fuel and $40 \%$ when using biodiesel and anamegators for fuel and oil, compared to standart diesel fuel.

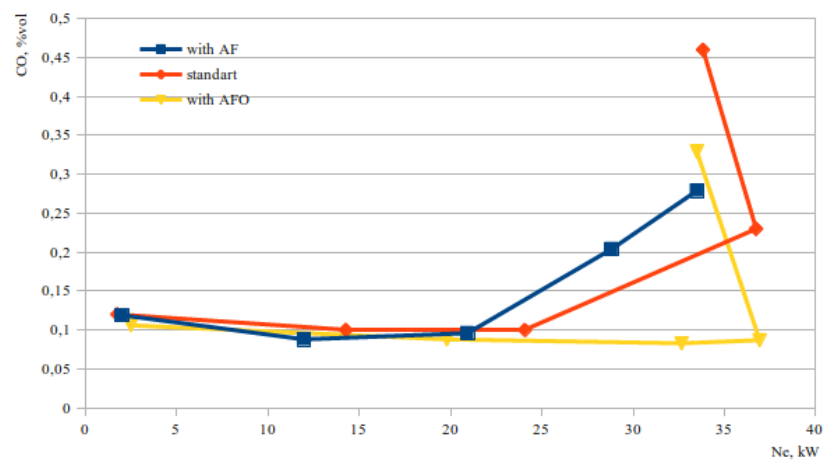

Fig. 5. Variation of carbon oxide in the exaust gases of diesel engine dependency from effective power:

with $\mathrm{AF}$ - mixed fuel with anamegator for fuels; standart - standart diesel fuel; with AFO - mixed fuel with anamegators for fuels and oils.

The variation of carbon dioxide is the opposite (figure 6). The whole reduction in free hydrocarbons and carbon monoxide results in a negligible increase in carbon dioxide. Its average increase for all regimes was $0,4 \%$ respectively when using biodiesel and anamegator for fuel to standard diesel and 7,5\% in the use of biodiesel and anamegators for fuel and for oil, together with standard diesel fuel. Obviously, the application of anamegators leads to a reduction of toxic emissions in exhaust gases from the diesel engine. Upon addition of the anemegator to the fuel, this reduction is increased.

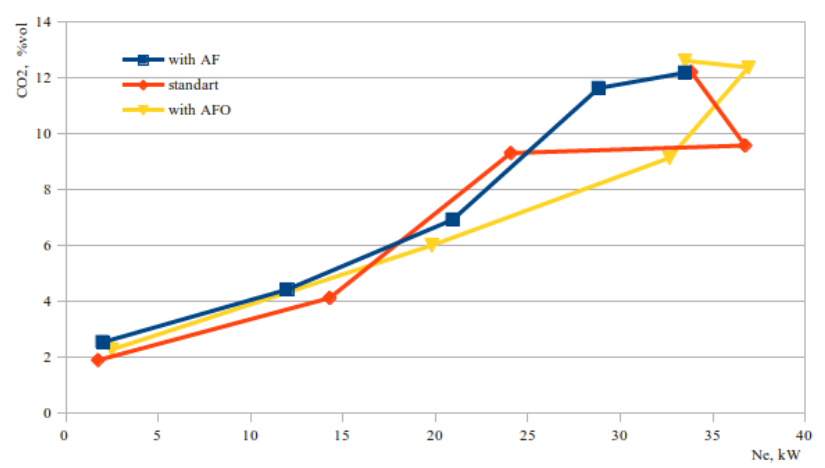

Fig. 6. Variation of carbon dioxide in the exaust gases of diesel engine dependency from effective power:

with $\mathrm{AF}$ - mixed fuel with anamegator for fuels; standart - standart diesel fuel; with AFO - mixed fuel with anamegators for fuels and oils.

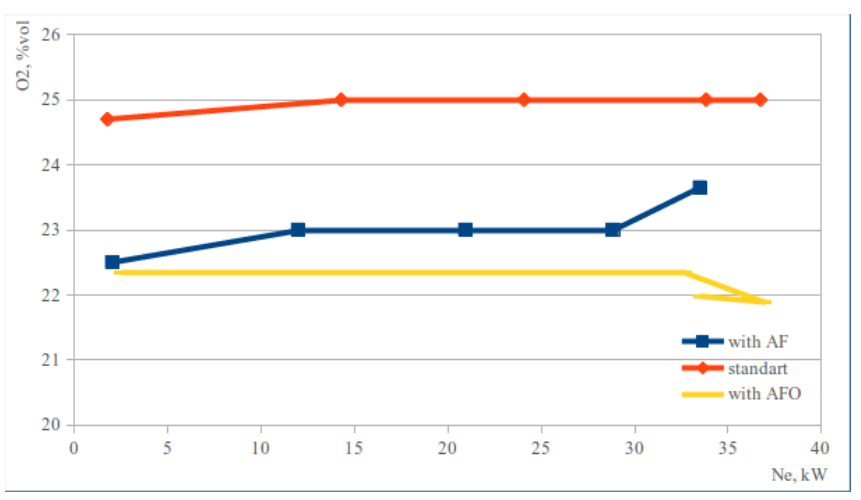

Fig. 7. Variation of excess oxygen in the exhaust gases of diesel engine dependency from effective power:

with $\mathrm{AF}$ - mixed fuel with anamegator for fuels; standart - standart diesel fuel; with AFO - mixed fuel with anamegators for fuels and oils.

As mentioned above and from figure 5 and 6 , it is apparent that the use of the anamegators results in the conversion of most of the $\mathrm{CO}$ into the $\mathrm{CO}_{2}$. This will reduce the amount of excess oxygen in the exhaust gas as shown in figure 7 . On the figure below clearly indicates the lower levels of this indicator. The decrease of this indicator is within 7,7\% when using the mixed fuel with anamegator for fuel and $11.1 \%$ when using the mixed fuel with anamegators for oil and for fuel.

The conversion of carbon monoxide into carbon dioxide, as well as the reduction of the oxygen content of the exhaust gas indicates the presence of stable combustion process in a diesel engine.

According to the manufacturer's instructions for the anamegators, they have no influence on the reduction of the nitrogen oxides, which is also is prominent from figure 8 . The average change in this indicator when using standard fuel and biodiesel with anamegators ranged from $8-10 \%$. This is normal considering the reduction of other toxic gases in the exhaust gases of the tested diesel engine. 


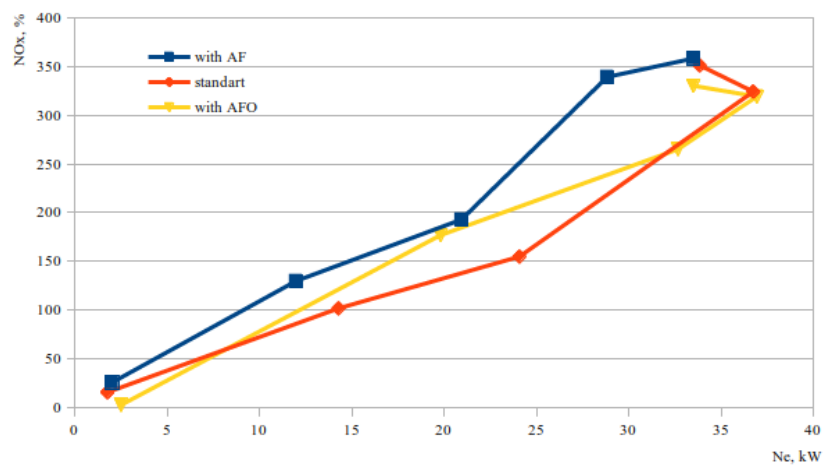

Fig. 8. Variation of nitrogen oxides in the exaust gases of diesel engine dependency from effective power:

with $\mathrm{AF}$ - mixed fuel with anamegator for fuels; standart - standart diesel fuel; with AFO - mixed fuel with anamegators for fuels and oils.

\section{Conclusion}

The use of biodiesel and anamegators for fuel and for oil leads to a reduction in fuel consumption and toxicity to the diesel engine.

Increasing fuel economy using $10 \%$ biodiesel and anamerges to fuel and oil with standard diesel fuel is within $22-23 \%$.

The reduction in the CO toxicity score is $22-40 \%$ when using biodiesel with anamegators for oil and for fuel blends to standard diesel fuel. This leads to a slight increase in the $\mathrm{CO}_{2}$ indicator.

The application of biodiesel andanamegators for fuel and for oil does not result in $\mathrm{NO}_{\mathrm{x}}$ reduction in the exhaust gases of a diesel engine.

\section{References}

1. G. Komitov, D. Kehayov, Proceeding of University of Ruse „Angel Kanchev“, book 4 - Transport and machine science, 51, 42 (2012).

2. G. Komitov, D. Kehayov, Scientific research of the Union of Scienists in Bulgaria-Plovdiv, series B, X, 33 (2013).

3. A. Ivanov, Machines, technologies, materials, 1, 54 (2007).

4. I. Mitkov, I. Ivanov, M. Dallev, Agricultural sciences, 20, 143 (2016).

5. I. Evtimov, R. Ivanov. Proceeding of University of Ruse „Angel Kanchev“, book 4 - Transport and machine science, 54, 121 (2015).

6. https://lukmir.com.ua/

7. H. Stanchev, E. Stankov, S. Babanov, A. Ali, Machines, technologies, materials, 10-11, 48 (2010). 